\title{
Annals of Biomedical Engineering 2020 Year in Review
}

\author{
Bethany Rowson and Stefan M. Duma \\ Department of Biomedical Engineering and Mechanics, Virginia Tech, Blacksburg, VA, USA
}

(Published online 23 February 2021)

The Annals of Biomedical Engineering (ABME) published its 48th issue in 2020. As the original journal of the Biomedical Engineering Society, ABME has a broad readership and aims to publish impactful research in all areas of Biomedical Engineering. ABME received its highest number of submissions in 2020, with 1254 submissions from 70 countries (Figs. 1, 2). As a result of increased submissions, the rejection rate has also increased to around $80 \%$. ABME accepted 255 papers in 2020 and published 225 in monthly issues. The monthly issues included three special issues on topics with growing interest in the biomedical engineering field: tissue engineering, biomaterials, and concussion biomechanics. The number of international submissions has continued to grow as well, with submissions from 70 countries last year compared with 58 countries in 2019 (Fig. 2). The top three countries in terms of submission numbers contributed nearly $50 \%$ of all submitted manuscripts: United States (23\%), China (14\%), and India (12\%). The following summarizes notable review papers and selected papers from each special issue.

\section{NOTABLE REVIEW PAPERS}

Donnely et al. reviewed tissue engineering-based breast reconstruction approaches..$^{5}$ Most studies used in vitro or animal models with scaffolds for supporting new tissue growth and migration. One successful human case series was reported. Scaffolds were either biological or synthetic, and some studies included cellular therapy with or without additional growth factors to enhance tissue regeneration. A current limitation that future research should address is improving vascularity for larger tissue volumes, potentially through adjusting tissue composition with cells and growth factors.

Address correspondence to Bethany Rowson, Department of Biomedical Engineering and Mechanics, Virginia Tech, Blacksburg, VA, USA. Electronic mail: browson@vt.edu
Zhao et al. provided a comprehensive review of the current state of robot-assisted fracture reduction. ${ }^{13}$ Current technologies lag behind robotics in other areas of orthopedic surgery. When optimized, surgical robots for fracture reduction can provide improved accuracy, less invasiveness, decreased radiation, and shorter recovery time. Current surgical robots are either serial, parallel, or hybrid robots. As technology progresses, robot-assisted fracture reduction could have broader applications outside the operating room. One potential use is telesurgery for situations where a patient cannot be transported to a hospital, including military operations or natural disasters.

Tejo-Otero et al. reviewed 3D printing techniques and their application to preoperative surgical planning. ${ }^{12}$ Printing methods and applications were reviewed, as well as ethical considerations involved with the technology. 3D-printed models of the affected organ can be used for visualization by both the surgeon and patient to better understand the planned procedure. Recent advances in printing technology have also allowed for printing tissue models with biofidelic material properties. With appropriate material properties, surgeons can use the models for practicing surgical techniques. Use of these models for preoperative surgical planning can shorten procedure times and enhance patient education.

Molinski et al. reviewed emerging technologies recently developed for signature-based molecular diagnostics. $^{7}$ Signature-based diagnostics use multiple biomarkers rather than a single marker for disease, which can improve the accuracy and specificity of a diagnostic test. The technology reviewed here combines microfluidics and micro-/nano-technologies with machine learning and imaging analysis for more accurate disease classification. A number of challenges exist in device design and integration, and data analysis that need to be addressed before these tools can be used clinically. However, preliminary work has shown great promise for signature-based diagnostics to greatly improve on currently available technology. 

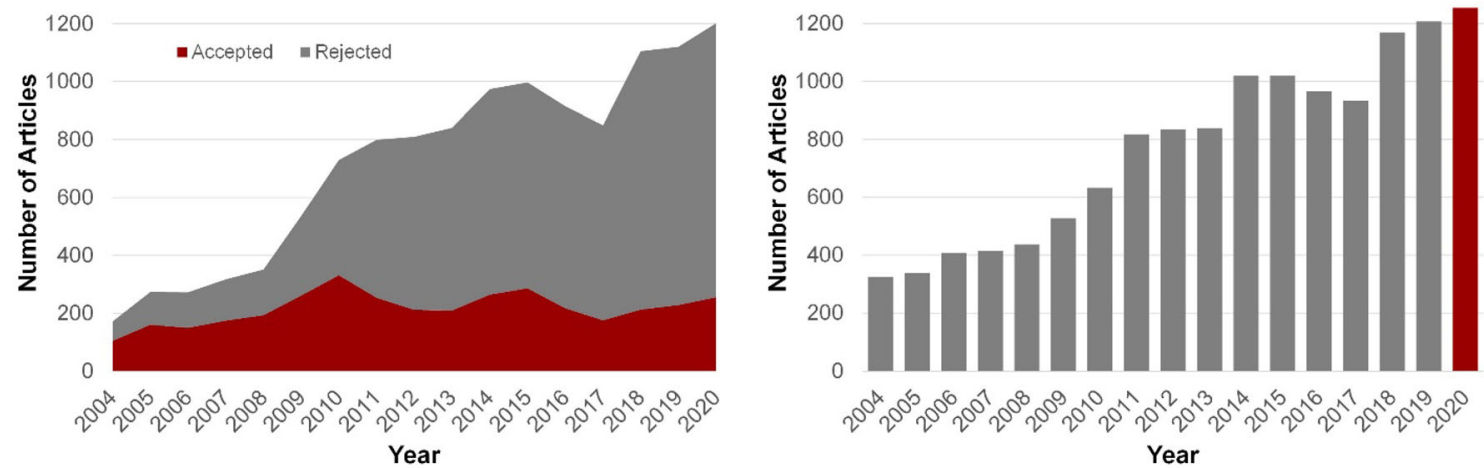

FIGURE 1. Total number of articles submitted per year (left) and proportion of articles accepted and rejected per year (right). Not all papers submitted in one year receive a decision in the same year, so the total number of articles in each plot are different.

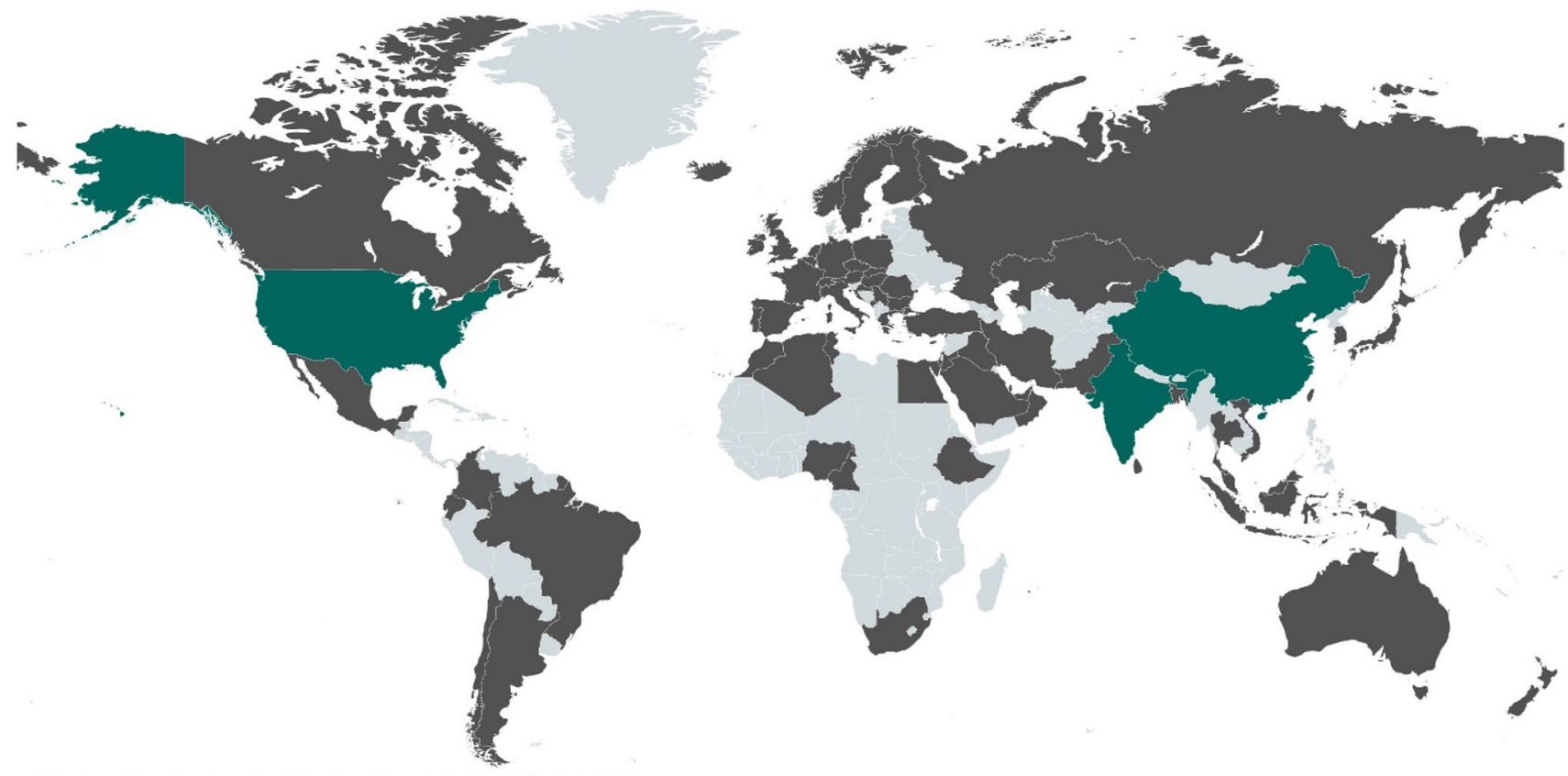

Countries that submitted articles to ABME in 2020

Countries with top 3 submission numbers in 2020

FIGURE 2. World map highlighting all countries that contributed submissions to ABME during 2020. The United States, China, and India had the highest numbers of submissions last year.

\section{SPECIAL ISSUES}

The first special issue published in 2020 was "Bioengineering and Enabling Technologies." ${ }^{\text {The special }}$ issue focused on the roles of biomaterials, techniques, cells, and bioactive factors used in tissue engineering. A prominent topic within the issue was fabrication of biologic scaffold materials for a variety of engineered tissue constructs. Padhi and Nain summarized the architecture, composition, and mechanical properties of extracellular matrix (ECM) within embryonic tissues, and fully developed tissue-specific ECM. ${ }^{9}$ ECM is an important driver of stem cell differentiation, so understanding the mechanisms involved and necessary ECM components can help guide tissue engineering techniques. The authors conclude that in order to optimize stem cell differentiation in vitro, biophysical cues present in tissue-specific ECM are needed. Talò et al. developed a novel automated bioreactor, the oscillating stretch-perfusion bioreactor (OSPB). ${ }^{11}$ The bioreactor can be used to culture multiple functional tendon grafts in parallel from cell-seeded, decellularized tendons. The device combines bidirectional perfusion with customizable uniaxial strain cycles. Following seven days of perfusion and stretching cycles, experimental samples showed viable cells, and 
improved production and organization of collagen matrix in comparison with static cultured constructs. The system is an effective tool for processing multiple customized tendon constructs, and could lead to improvements in surgical repair of tendon defects.

The second 2020 special issue was "Biomaterials-Engineering Cell Behavior." This issue focused on cellular behavior and how it is applied to engineering biomaterials. Biomaterials have broad applications in tissue engineering and drug delivery, with the most recent advances explored in these articles. Cramer and Badylak reviewed production methods used for biologic scaffold materials and the differences in clinical outcomes associated with each method. ${ }^{3}$ Bioscaffolds are derived from mammalian ECM for injured tissue repair, and have advantages for tissue regeneration including release of bioactive molecules, recruitment of stem cells, and modulation of the immune response. However, the clinical outcome following tissue repair can vary greatly depending on the source of the tissue the scaffold was derived from, the decellularization method, and processing of the scaffold after decellularization. D'Costa et al. summarized the development and applications of 3D tissue culture systems. ${ }^{4}$ These culture systems overcome many limitations of $2 \mathrm{D}$ cultures by more accurately modeling in vivo tissue environments. The use of biomaterials in 3D cultures has contributed to development of physiologically relevant tissue architectures and environments containing multiple cell types. These models have been used to study developmental mechanisms of both healthy and diseased tissues, and can be used for discovery of novel therapeutics.

The final special issue of 2020, "Concussion Biomechanics in Football," was published in November. ${ }^{10}$ This issue focused on the most recent advances in concussion biomechanics in American football players. Development and evaluation of head impact sensors, and injury reduction strategies including behavioral modification and helmet evaluation were highlighted. Liu et al. performed laboratory validation of five commonly used instrumented mouthguards for measuring head impact kinematics. ${ }^{6}$ All mouthguards had average errors less than $13 \%$ for peak rotational acceleration, peak rotational velocity, and brain injury criteria calculated from head kinematics. Brain strain was also determined with a convolutional neural network-based brain model for mouthguards that captured a long enough time window during impact. Instrumented mouthguards have shown promise for collecting more accurate head impact kinematics than alternative sensors in sports because of the rigid coupling of the upper dentition to the skull. Bailey et al. compared laboratory impact performance of National Football League (NFL) helmets to on-field perfor- mance. ${ }^{2}$ Laboratory performance was summarized for a series of impacts to each helmet through a linear combination of head injury criterion (HIC) and a brain strain correlate metric (DAMAGE). On-field performance was quantified by collecting helmet model usage, player participation, and concussion data from NFL players. There was a positive correlation between laboratory data and on-field performance, indicating that helmets that reduce head kinematic measures in laboratory impacts will have a lower concussion rate on the field.

As ABME submission numbers continue to grow, so does the quality and impact of the papers published. We thank our authors for submitting their important work, and our reviewers for volunteering their time and insight to refine the papers published. The journal would not be possible without these valuable contributions.

\section{REFERENCES}

${ }^{1}$ Auguste, D. ABME special issue editorial: engineering cell behavior. Ann. Biomed. Eng. 48:1863, 2020.

${ }^{2}$ Bailey, A. M., T. L. McMurry, J. M. Cormier, J. R. Funk, J. R. Crandall, C. D. Mack, B. S. Myers, and K. B. Arbogast. Comparison of laboratory and on-field performance of American football helmets. Ann. Biomed. Eng. 48:2531-2541, 2020.

${ }^{3}$ Cramer, M. C., and S. F. Badylak. Extracellular matrixbased biomaterials and their influence upon cell behavior. Ann. Biomed. Eng. 48:2132-2153, 2020.

${ }^{4}$ D'Costa, K., M. Kosic, A. Lam, A. Moradipour, Y. Zhao, and M. Radisic. Biomaterials and culture systems for development of organoid and organ-on-a-chip models. Ann. Biomed. Eng. 48:2002-2027, 2020.

${ }^{5}$ Donnely, E., M. Griffin, and P. Butler. Breast reconstruction with a tissue engineering and regenerative medicine approach (systematic review). Ann. Biomed. Eng. 48:925, 2020.

${ }^{6}$ Liu, Y., A. G. Domel, S. A. Yousefsani, J. Kondic, G. Grant, M. Zeineh, and D. B. Camarillo. Validation and comparison of instrumented mouthguards for measuring head kinematics and assessing brain deformation in football impacts. Ann. Biomed. Eng. 48:2580-2598, 2020.

${ }^{7}$ Molinski J., A. Tadimety, A. Burklund and J. X. Zhang. Scalable signature-based molecular diagnostics through on-chip biomarker profiling coupled with machine learning. Ann. Biomed. Eng. 1-23, 2020.

${ }^{8}$ Opara, E. C. Bioengineering and enabling technologies: ABME special issue editorial. Ann. Biomed. Eng. 48:14451450, 2020.

${ }^{9}$ Padhi, A., and A. S. Nain. ECM in differentiation: a review of matrix structure, composition and mechanical properties. Ann. Biomed. Eng. 48:1071-1089, 2020.

${ }^{10}$ Rowson, B., and S. M. Duma. Special issue on concussion biomechanics in football. Ann. Biomed. Eng. 48:2495-2496, 2020.

${ }^{11}$ Talò, G., D. D'Arrigo, S. Lorenzi, M. Moretti, and A. B. Lovati. Independent, controllable stretch-perfusion biore- 
actor chambers to functionalize cell-seeded decellularized tendons. Ann. Biomed. Eng. 48:1112-1126, 2020.

${ }^{12}$ Tejo-Otero, A., I. Buj-Corral, and F. Fenollosa-Artés. 3D printing in medicine for preoperative surgical planning: a review. Ann. Biomed. Eng. 48:536-555, 2020.

${ }^{13}$ Zhao, J.-X., C. Li, H. Ren, M. Hao, L.-C. Zhang, and P.-F. Tang. Evolution and current applications of robot-assisted fracture reduction: a comprehensive review. Ann. Biomed. Eng. 48:203-224, 2020.

Publisher's Note Springer Nature remains neutral with regard to jurisdictional claims in published maps and institutional affiliations. 